\title{
Primary pigmented nodular adrenocortical disease
}

\author{
Harris Abdullah Ngow ${ }^{1}$, Wan Mohd Nowalid Wan Khairina ${ }^{2}$ \\ ${ }^{1}$ International Islamic University of Malaysia/Hospital Tengku Ampuan Afzan, Kulliyyah Of Medicine, Jalan Hospital, Pahang, \\ Malaysia \\ ${ }^{2}$ Ministry of Health, Malaysia
}

\begin{abstract}
Primary pigmented nodular adrenocortical disease (PPNAD) is a rare adrenal tumour causing corticotrophin-independent Cushing's syndrome. It occurs mainly in children and young adults. The histological examination is characterised by small pigmented micronodules on the adrenal cortex. The diagnosis is most often seen in patients with Carney Complex, but it can also occur in isolation. We report a case of Carney Complex that was referred for adrenalectomy. The procedure was uneventful and the patient was well at discharge. The adrenal pathology showed numerous black nodules measuring less than $2 \mathrm{~mm}$ in diameter. This feature was pathognomonic of primary pigmented nodular adrenocortical disease. (Pol J Endocrinol 2011; 62 (3): 268-270)
\end{abstract}

Key words: PPNAD, Carney complex, adrenal gland, tumour

\section{Introduction}

Primary pigmented nodular adrenocortical disease (PPNAD) is the main endocrine manifestation of Carney Complex. , which is a multiple neoplasia syndrome caused by PRKAR1A gene mutation. The syndrome is characterised by cutaneous lentigines, myxoma, schwannomas and endocrinopathy [9]. It was first described in 1985 by J Aidan Carney and co-workers at the Mayo Clinic [2]. It has rarely been reported in South East Asia. Indeed, we can only find a single report on this condition [8].

We present the case of a young woman who was referred for bilateral adrenalectomy. Her adrenal histology revealed multiple pigmented micronodules that were suggestive of PPNAD. Genomic study was suggested, but the patient refused it.

\section{Case report}

The patient was a 23 year-old Chinese woman who worked as an accounts clerk. She was referred for bilateral adrenalectomy by a private endocrinologist. She had been on treatment for hypertension for the past three years. Her blood pressure was well controlled with atenolol and nifedipine. She also complained of amenorrhoea for six months prior to this referral. She had noticed increasing numbers of freckles over her face and arms. She had no history of hirsutism or virilisation. She did not complain of proximal myopathy, weight gain or abdominal striae. Two of her elder sisters had been diagnosed with Cushing's syndrome and had undergone bilateral adrenalectomy. Her eldest sister died of acute stroke at the age of 33. She denied taking traditional or over-the-counter medicine.

On examination, she had no cushingoid features. Her blood pressure was 130/85 mmHg. Her pulse rate was 76 beats/minute. There was an absence of the stigmata of Cushing's syndrome such as truncal obesity, paper-thin skin, abdominal striae, buffalo's hump, hyperpigmentation or proximal myopathy. Multiple freckles were noted on the face and arms. The praecordium examination was normal and a murmur was not present. The examination of the breast was normal. The chest and abdominal examinations were unremarkable. The neurological examination was normal without any localising signs. Fundoscopy examination did not reveal papilloedema.

Her haematological investigations, including haemoglobin, leucocytes count, and platelet count, were normal. Serum electrolytes, glucose, creatinine, liver function test, fasting serum lipid, serum prolactin and CA-125 were all normal. The early morning plasma cortisol was $329 \mathrm{nmol} / \mathrm{L}$. However, the level of early morning plasma cortisol had been more than $1,000 \mathrm{nmol} / \mathrm{L}$ when it was performed previously by the

Harris Abdullah Ngow, Kulliyah Of Medicine, International Islamic University, P.O. Box 141, Kuantan, Pahang Darul Makmur, Malaysia,

Tel: 6-09-513 2797, fax: 6-09-513 3615, e-mail: harrisngow@gmail.com 

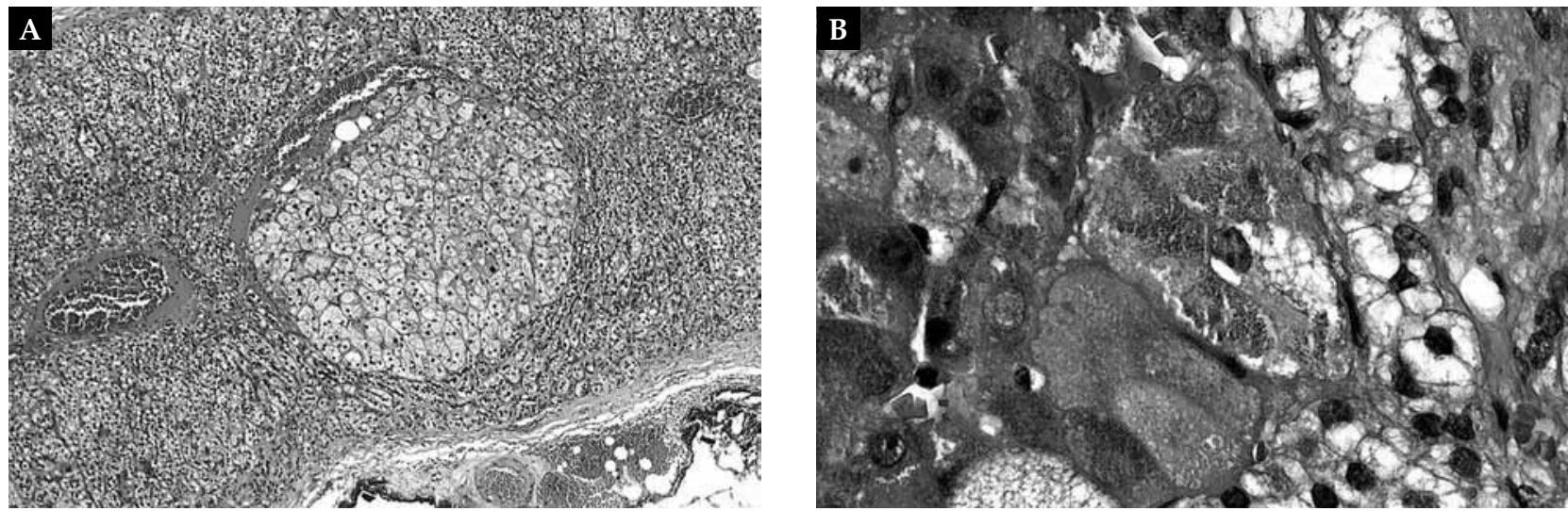

Figure 1AB. $A$. $(H \in E) \times 10$. B. $(H \in E) \times 40$. The pigmented nodules that found in the adrenal cortex of this patient. The adrenal gland is hyperplastic with a macronodule ( $6 \mathrm{~mm}$ in diameter) seen. Numerous micro-nodules were seen and there was uniform proliferation of cells with foci of cytoplasmic clearing and arranged in compact nests

private endocrinologist. The random plasma ACTH was undetectable (less than $10 \mathrm{pg} / \mathrm{mL}$ ). The low dose dexamethasone suppression test failed to suppress the adrenal cortical activity. A similar finding was found with high dose dexamethasone suppression test. The echocardiogram showed good left ventricular systolic function with normal cardiac chambers and an absence of atrial myxoma. Abdominal ultrasound showed multiple cystic lesions on both ovaries. CT scan of the abdomen revealed multiple bilateral micronodular adrenal hyperplasias. MRI of the brain showed a non-functioning pituitary incidentaloma.

The immediate clinical diagnosis was adrenocorticotrophic independent Cushing's syndrome secondary to bilateral micronodular adrenal hyperplasia. She underwent adrenalectomy with partial re-implantation of the adrenal tissue over the left forearm. The procedure was successful and she was discharged in a good condition after five days. She opted to be followed up by her private endocrinologist, and was discharged from our care. Her adrenal histological examination was reported as primary pigmented nodular adrenocortical disease. The hallmark of the histological examination is the presence of multiple black nodules less than $2 \mathrm{~mm}$ in diameter. (Figures $1 \mathrm{AB}, 2$ ). A further genomic study to detect PRKAR1A was discussed, but the patient refused the test.

\section{Discussion}

Carney Complex is a familial multiple endocrine neoplasia syndrome that is characterised by cardiac and cutaneous myxoma, lentigines and endocrinopathy. It is an autosomal dominant disease. The disease has a familial pattern in approximately $50 \%$ of patients, while in others it appears sporadically [4]. The clinical diagnosis

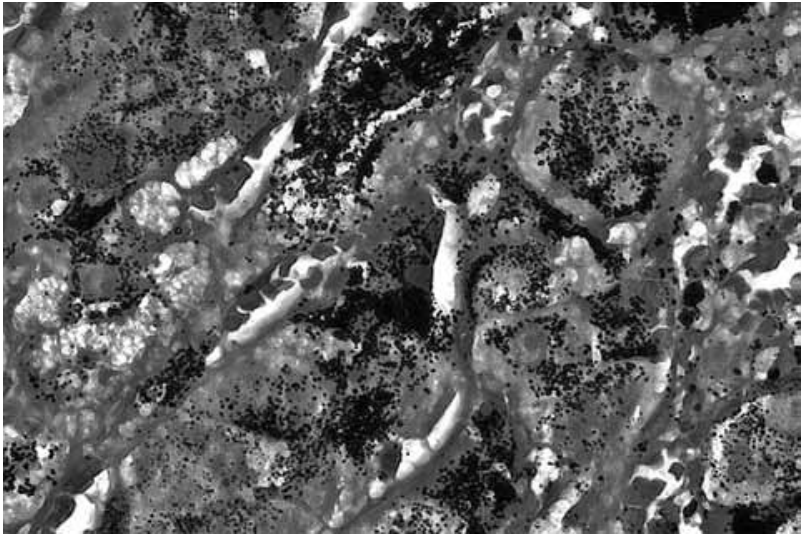

Figure 2. Synaptophysin immunostain. The numerous blackish pigments found on the adrenal cortex of this patient with primary pigmented nodular adrenocortical disease

is made when there is at least one characteristic clinical finding associated with the disease in the presence of a family history of Carney Complex. In the absence of family history, there should be at least two characteristic clinical findings [10].

PPNAD is a rare cause of adrenocorticotrophic hormone independent Cushing's syndrome. Adrenocorticotrophic hormone independent Cushing's syndrome is observed in $25-30 \%$ of patients with Carney Complex [8]. It has a predilection towards children and young adults. The peak incidence in adults is in their 20s, as was our patient. This tumour is very rarely found in children under the age of four and rarely diagnosed in adults after the age of 40 [7]. It is estimated that the diagnosis is made in no more than $1 \%$ of patients with Cushing's syndrome [1]. The name derives from the macroscopic appearance of the adrenals that is characterised by small pigmented micronodules in the adrenal cortex. The disease usually involves bilateral adrenals. The 
diagnosis of PPNAD can be made in an asymptomatic patient by familial screening or personal screening for features associated with Carney Complex.

Our patient was diagnosed based on her strong family history as well as increasing numbers of lentigines on her face and arms. The low dose and high dose dexamethasone suppression test confirmed the diagnosis of ACTH independent Cushing's syndrome. In addition, her abdominal CT scan further strengthened the diagnosis of PPNAD.

The disease can present in several ways. Our patient presented with increasing cutaneous lentigines over her face and arms. In addition, she had a strong family history of features related to Carney Complex. Therefore, she was screened for PPNAD confirmed by HDDST, and adrenal hyperplasia was also visualised on her abdominal CT scan . The diagnosis of Cushing's syndrome in PPNAD is often difficult because hypercortisolism may progress slowly over many years or may be cyclical [5, 6]. Cyclical hypercortisolism has been described in some patients with PPNAD. This could explain the absence of cushingoid features in our patient.

Cardiac and other cutaneous myxomas, spotty velvety cutaneous pigmentation and endocrine tumours are the main characteristics of this disease. Cardiac myxoma is an important feature. Unrecognised cardiac myxoma may be the cause of sudden cardiac death due to thromboembolism. Although the echocardiographic examination was normal, our patient requires close monitoring for the development of this tumour. Her eldest sister died due to acute stroke which could have been the result of a thrombotic event from an undetected cardiac myxoma. Cutaneous pigmentation is the commonest manifestation of the disease [10]. Lentigines are seen in most patients, and this characteristic manifestation can be used to make the definitive diagnosis
[5]. Other common endocrine tumours include pituitary adenomas, thyroid adenomas and carcinoma, testicular tumours and ovarian tumours $[5,10]$.

In conclusion, the manifestations of Carney Complex can vary between patients. Lentigines are the main manifestation. The recognition of adrenal Cushing's syndrome can be difficult in some patients with cyclical hypercortisolism. Patients should also be screened for cardiac myxoma periodically to avoid sudden unexpected death. In most cases, removal of the bilateral adrenal glands is required to demonstrate an apparent cure for the disease. Long term follow-up of this patient is mandatory to detect occurrence of other associated tumours. Awareness of the various components of the disease will help identify affected patients, as will screening of their first degree relatives.

\section{References}

1. Berherat J. Carney complex (CNC). Orphanet J Rare Dis 2006; 1: 21.

2. Carney JA,Gordon H, Carpenter PC, Shenoy BV, Go VL. The complex of myxomas, spotty pigmentation and endocrine overactivity. Medicine (Baltimore) 1985; 64: 270-283.

3. Carney JA, Young WF. Primary pigmented nodular adrenocortical disease and its associated conditions. The Endocrinologist 1992; 2: 6-21.

4. Courcoutsakis NA, Chow SK, Shawker TH, Carney JA, Stratakis CA. Syndrome of spotty skin pigmentation, myxomas, endocrine overactivity and schwannomas 9carney complex): breast imaging findings. Radiology 1997; 205: 221-227.

5. Groussin L, Jullian E, Perlemoine K et al. Mutations of the PRKAR1A gene in Cushing's syndrome due to sporadic primary pigmented nodular adrenocortical disease. J Clin Endocrinol Metab 2002; 87: 4324-4329.

6. Gunther DF, Bourdeau I, Matyakhina L et al. Cyclical Cushing's syndrome presenting in infancy: an early form of primary pigmented nodular adrenocortical disease, or a new entity? J Clin Endocrinol Metab 2004; 89: 3173-182.

7. Lionel G, Laure C, Fernande RC, Eric J, Jerome B. Adrenal pathophysiology: Lessons from the Carney complex. Horm Res 2005; 64: 132-139.

8. Norhaliza MA, Zanariah H, Masni M. Carney complex: Cardiac myxoma and Cushing's syndrome. JAFES 2009; 24: 28.

9. Stratakis CA, Kirschner LS, Carney JS. Clinical and molecular features of the Carney complex: diagnosis criteria and recommendations for patient evaluation. J Clin Endocrinol Metab 2001; 86: 4041-4046.

10. Wilkes D, McDermott DA, Basson CT. Clinical phenotypes and molecular genetic mechanisms of Carney complex. Lancet Oncol 2005; 6 :501-508. 